DOI: 10.12957/demetra.2016.18457

\title{
Desnutrição na admissão, permanência hospitalar e mortalidade de pacientes internados em um hospital terciário
}

\section{Malnutrition on admission, length of hospital stay and mortality of hospitalized patients in a tertiary hospital}

\author{
Vivian Plaça Teixeiral \\ Renata Costa de Miranda' \\ Deise Regina Baptista' \\ 'Universidade Federal do Paraná, Setor \\ de Ciências da Saúde, Departamento de \\ Nutrição. Curitiba-PR, Brasil. \\ Correspondência / Corrrespondence \\ Vivian Plaça Teixeira \\ E-mail: vivi.teixeira0@gmail.com
}

\section{Resumo}

Objetivo: Determinar a frequência de desnutrição na admissão de pacientes para internação, verificando possíveis influências relacionadas ao tempo de permanência hospitalar e à mortalidade. Métodos: O estudo possui caráter observacional transversal retrospectivo, com coleta e análise de dados dos prontuários dos pacientes admitidos para internação na Clínica Médica do Hospital de Clínicas da UFPR no período de janeiro a julho de 2013. Para identificação do risco nutricional, foi utilizada a triagem nutricional Nutritional Risk Screening-NRS 2002. Pacientes com risco nutricional passaram por avaliação nutricional, de acordo com o protocolo do Serviço. Resultados: A amostra de 148 pacientes foi composta majoritariamente por homens adultos. A doença mais frequente foi a HAS, tanto para a amostra total como para os desnutridos. As doenças cardiovasculares, do trato geniturinário, neoplásicas, HAS e outras foram mais frequentes em idosos; e as doenças autoimunes, em adultos. A média de doenças da amostra foi de 2,22 $\pm 1,16$, e os idosos possuíam maior número de doenças associadas. Pacientes com risco nutricional representaram $58,8 \%$ da amostra; entre estes, $54,7 \%$ eram desnutridos, $0,7 \%$ tinham peso normal, $1,4 \%$ apresentavam sobrepeso e 2,1\% eram obesos. O tempo médio de permanência hospitalar dos pacientes desnutridos foi de 14,05 $\pm 11,87$, e dos não desnutridos 15,43 \pm 13,81 dias, não houve diferença estatística. O índice de mortalidade da amostra total foi de $5,4 \%$, entre estes, 25\% estavam desnutridos. Conclusão: Constatou-se alta frequência de desnutrição, porém não houve relação com o prolongamento da permanência hospitalar e com a mortalidade dos pacientes.

Palavras-chave: Desnutrição; Tempo de Internação; Mortalidade; Pacientes Internados; Admissão do paciente. 


\section{Abstract}

Objective: To determine the frequency of malnutrition on admission of patients and verify the influences related to the length of hospital stay and mortality. Methods: This retrospective cross-sectional observational study was made up of data collection and medical records analysis of inpatients on the Clínica Médica do Hospital de Clínicas da UFPR, from January to July 2013. In order to identify the nutritional risk, the Screening Nutritional Risk Screening - 2002 was used. Patients at nutritional risk were submitted to nutritional evaluation, in accordance to the Protocol Service. Results: The sample counted with 148 patients, mainly adult males. The most prevalent disease in the overall sample and among the malnourished patients was hypertension. Cardiovascular disease, genitourinary disorders, neoplasms, hypertension and other diseases were most prevalent in the elderly patients, and autoimmune diseases were the most prevalent in adults. The average of diseases in our sample was $2.22 \pm 1.16$, and elderly patients had the higher number of associated diseases. Patients at nutritional risk were $58.8 \%$ of the sample; among these, $54.7 \%$ were malnourished, $0.7 \%$ was normal weight, $1,4 \%$ was overweight and $2.1 \%$ was obese. The length of hospital stay average was $14.05 \pm 11.87$ days among malnourished patients, and $15.43 \pm 13.81$ days among non malnourished, without statistical difference. The mortality rate of the sample was $5.4 \%$, among which $25 \%$ were malnourished. Conclusion: It was found high frequency of malnutrition but not related to the prolonged hospital stay and mortality of patients.

Key words: Malnutrition; Length of Stay; Mortality; Inpatients; Patient Admission.

\section{Introdução}

Entre as diversas condições clínicas relacionadas ao estado nutricional, encontra-se a desnutrição, que, segundo Caldwell et al., é "um estado mórbido secundário a uma deficiência ou excesso relativo ou absoluto, de um ou mais nutrientes essenciais, que se manifesta clinicamente ou é detectada por meio de testes bioquímicos, antropométricos, topográficos ou fisiológicos."

A etiologia da desnutrição detectada na admissão hospitalar pode ser primária, decorrente da ingestão inadequada e insatisfatória de nutrientes, consequência de condições socioeconômicas precárias que não garantem a reposição energético-proteica e dos demais nutrientes necessários; ou secundária, decorrente de fatores intrínsecos, pelo impacto de doença. 
As principais causas da desnutrição associada à doença são a deficiência na ingestão alimentar, efeitos de medicamentos, redução da absorção de macro e micronutrientes, alteração das necessidades nutricionais e do gasto energético. ${ }^{3-5}$

Na década de setenta, o estudo realizado por Bollet e Owens ${ }^{6}$ se destacou pela elevada prevalência de desnutrição em pacientes hospitalizados por diversas doenças. Mesmo após quarenta anos, estudos atuais ${ }^{7-9}$ vêm corroborando resultados similares e demonstrando que a desnutrição hospitalar continua a ser um problema atual e recorrente, independentemente do nível de desenvolvimento do país.

No Brasil, a partir do Inquérito Brasileiro de Avaliação Nutricional Hospitalar (IBRANUTRI), estudo realizado com quatro mil pacientes de hospitais distribuídos por 12 estados do país, revelou que $48 \%$ dos pacientes estavam desnutridos, sendo que destes, 33,2\% já possuíam esta condição nos primeiros dois dias, constatando que grande parte dos pacientes desnutridos já possuía um estado nutricional debilitado no início da internação. ${ }^{10}$

Pacientes internados podem desenvolver desnutrição após a admissão hospitalar, assim como até $70 \%$ dos desnutridos inicialmente pioram seu estado nutricional ao longo da internação, aumentando o risco de infecções, mortalidade, tempo de permanência hospitalar e custos para o hospital e o sistema de saúde. ${ }^{11-14}$

A resposta ao estresse gerado pela doença ou por outras causas da desnutrição, como a ingestão diária alimentar dimiuída em longo prazo, faz com que o organism utilize reservas do tecido muscular e adipose para produzir energia, levando a mudanças na composição corporal, redução da funcionalidade, alterações sanguíneas, nos demais órgãos e sistemas e a um estado metabólico frágil. ${ }^{5,15}$

Este estudo tem como objetivo determinar a frequência de desnutrição na admissão hospitalar e correlacionar a desnutrição com o tempo de permanência hospitalar e a mortalidade de pacientes clínicos.

\section{Métodos}

O presente estudo possui caráter observacional transversal retrospectivo e foi realizado a partir de 205 prontuários de pacientes adultos e idosos, de ambos os sexos, admitidos para internação entre janeiro e julho de 2013, na Clínica Médica Feminina e Masculina do Hospital de Clínicas da Universidade Federal do Paraná.

O estudo faz parte do projeto de pesquisa intitulado "Alterações nutricionais durante a internação: perfil de um hospital público terciário”, aprovado pelo Comitê de Ética em Pesquisa 
com Seres Humanos do Hospital de Clínicas da Universidade Federal do Paraná, com parecer de número 880.451 .

Os dados coletados dos prontuários foram preenchidos por nutricionistas do Serviço, por meio do protocolo de atendimento nutricional previamente definido, constituído para registro da identificação do paciente (idade e sexo), informações médicas (diagnóstico médico, data da internação e da alta hospitalar e confirmação de óbito ou não) e Triagem Nutricional (Nutritional Risk Screening-NRS $2002^{16}$ ), realizada nas primeiras 48 horas da admissão do paciente.

A Triagem Nutricional (NRS-2002) é recomendada pela American Society for Parenteral and Enteral Nutrition (ASPEN), sendo composta por quatro questões objetivas sobre o paciente: índice de massa corporal (IMC), perda de peso não intencional nos últimos três meses, ingestão alimentar reduzida na última semana e presença de doença grave ou estado crítico. Por meio dessas informações, permite identificar características associadas a problemas nutricionais e a possibilidade do risco nutricional..$^{16} \mathrm{~A}$ i dentificação do risco nutricional foi classificada em: sem risco nutricional ou com risco nutricional.

Caso o risco nutricional fosse identificado, o paciente era submetido a uma avaliação nutricional constituída por avaliação antropométrica, bioquímica, nutricional global subjetiva e dietética, de acordo com o protocolo do próprio hospital, com o objetivo de diagnosticar seu estado nutricional. O diagnóstico do estado nutricional foi classificado em: desnutrição grave, desnutrição moderada, desnutrição leve, eutrofia, sobrepeso, obesidade grau l, obesidade grau ll ou obesidade grau lll. Os pacientes diagnosticados com desnutrição grave, moderada e leve foram incluídos no grupo dos desnutridos, e os demais no grupo dos não desnutridos.

Os diagnósticos médicos relatados nos prontuários de admissão dos pacientes foram classificados em 14 categorias de doenças: autoimunes, cardiovasculares, endócrinas, do trato geniturinário, hipertensão arterial sistêmica, hematológicas, imunológicas, neoplásicas, neurológicas, respiratórias, reumáticas, do sistema nervoso, do trato gastrointestinal e outras. Na categoria "outras", foram incluídas doenças e condições clínicas que não se encaixaram nas outras 13 categorias de doenças, evitando mais subdivisões, devido à grande diversidade de doenças.

Foram excluídos 57 prontuários que apresentavam dados incompletos de idade, diagnóstico médico e/ou diagnóstico nutricional dos pacientes, impossibilitando a análise dos dados.

Os dados coletados foram analisados estatisticamente no programa Statistical Package for the Social Science (SPSS 20.0). O teste Mann-Whitney foi utilizado com a finalidade de verificar a diferença entre médias de dados não paramétricos; os testes Qui-quadrado e Exato de Fischer foram utilizados para comparar as distribuições de frequência; e o teste ANOVA, para comparar mais de dois grupos. O intervalo de confiança assumido foi de $95 \%$. 


\section{Resultados}

Dos 148 pacientes incluídos no estudo, 59,5\% eram do sexo masculino e 40,5\% do sexo feminino, com idade média de 52,59 \pm 17,48 anos. Os adultos representaram esta população em $64,2 \%$ e os idosos em 35,8\%, com idade média de 41,94 \pm 11,33 e 71,68 \pm 7,30 anos, respectivamente (tabela 1). A doença mais frequente diagnosticada nos prontuários dos pacientes foi a HAS $(44,6 \%)$ e a de menor frequência foi o grupo das doenças neurológicas (0,7\%) (tabela 1).

Tabela 1. Caracterização demográfica e clínica da população estudada na Clínica Médica Feminina e Masculina do Hospital de Clínicas da Universidade Federal do Paraná. Curitiba-PR, 2013.

\begin{tabular}{|c|c|c|c|c|}
\hline & Adultos & Idosos & $p$ & Total \\
\hline Total pacientes $(\%)$ & 64,20 & 35,80 & & 100 \\
\hline \multirow{2}{*}{$\operatorname{Sexo}(\%)$} & Feminino: 40 & Feminino: 41,5 & & \\
\hline & Masculino: 60 & Masculino: 58,5 & & 100 \\
\hline \multirow{2}{*}{ Idade $(x \pm \mathrm{DP})$} & $41,94 \pm$ & $71,68 \pm$ & & $52,59 \pm$ \\
\hline & 11,33 & 7,30 & & 17,48 \\
\hline \multicolumn{5}{|l|}{ Categoria de doenças (\%) } \\
\hline Autoimunes $^{\mathrm{a}}$ & 10,5 & 0 & $0,01 *$ & 6,8 \\
\hline Cardiovasculares $^{\mathrm{a}}$ & 5,3 & 24,5 & $0,00^{*}$ & 12,2 \\
\hline Endócrinas $^{\mathrm{a}}$ & 33,7 & 38,6 & 0,47 & 35,8 \\
\hline Trato geniturinário ${ }^{a}$ & 13,7 & 37,7 & $0,00^{*}$ & 22,3 \\
\hline $\mathrm{HAS}^{\mathrm{a}}$ & 37,9 & 56,6 & $0,02 *$ & 44,6 \\
\hline Hematológicas $^{\mathrm{a}}$ & 13,7 & 11,3 & 0,68 & 12,8 \\
\hline Imunológicas $^{\mathrm{b}}$ & 1,1 & 1,9 & 1,00 & 1,4 \\
\hline Neoplásicas $^{\mathrm{a}}$ & 9,5 & 22,6 & $0,02 *$ & 14,2 \\
\hline Neurológicas $^{\mathrm{b}}$ & 1,1 & 0 & 1,00 & 0,7 \\
\hline Outras $^{\mathrm{a}}$ & 24,2 & 34 & $0,02 *$ & 27,7 \\
\hline Respiratórias $^{\mathrm{a}}$ & 11,6 & 17 & 0,35 & 13,5 \\
\hline Reumáticas $^{\mathrm{a}}$ & 5,3 & 11,3 & 0,17 & 7,4 \\
\hline Sistema Nervoso $^{\mathrm{b}}$ & 6,3 & 0 & 0,08 & 4,1 \\
\hline Trato gastrointestinal ${ }^{\mathrm{a}}$ & 23,2 & 13,2 & 0,14 & 19,6 \\
\hline
\end{tabular}

Teste Qui-quadrado

bTeste Exato de Fischer

HAS $=$ Hipertensão arterial sistêmica; $\mathrm{DP}=$ Desvio padrão

"Diferença significativa $(\mathrm{p}<0,05)$ 
As categorias de doenças cardiovasculares, do trato geniturinário, neoplásicas, HAS e outras apresentaram diferença significativa entre adultos e idosos, sendo mais prevalentes em idosos $(\mathrm{p}<0,0001, \mathrm{p}<0,0001, \mathrm{p}=0,02, \mathrm{p}=0,02, \mathrm{p}=0,02$, respectivamente). A categoria das doenças autoimunes também apresentou diferença significativa, sendo mais frequente em adultos $(\mathrm{p}=0,01)$. As demais doenças não apresentaram diferença significativa em relação à faixa etária (tabela 1).

Em relação à detecção de risco nutricional e à avaliação nutricional dos pacientes ao serem internados, foram diagnosticados 41,2\% sem risco nutricional e 58,8\% com risco nutricional. A frequência total de desnutrição dos pacientes avaliados foi de 54,7\%. Entre os idosos, 62,3\%, e entre os adultos, $50,5 \%$ estavam desnutridos. Não houve diferença estatística entre a faixa etária e a presença de desnutrição $(\mathrm{p}=0,16)$ (tabela 2).

Tabela 2. Estado nutricional e prevalência geral de desnutrição da população estudada na Clínica Médica Feminina e Masculina do Hospital de Clínicas da Universidade Federal do Paraná. Curitiba-PR, 2013

\begin{tabular}{lccc}
\hline \multicolumn{1}{c}{ Estado nutricional } & Adultos $(\%)$ & Idosos $(\%)$ & Total (\%) \\
\hline Sem risco & 47,7 & 30,2 & 41,2 \\
Com risco & 52,3 & 69,8 & 58,8 \\
Desnutrição grave & 8,4 & 15,1 & 10,8 \\
Desnutrição moderada & 17,9 & 15,1 & 16,9 \\
Desnutrição leve & 24,2 & 32,1 & 27 \\
Eutrofia & 0 & 1,9 & 0,7 \\
Sobrepeso & 0 & 3,8 & 1,4 \\
Obesidade grau I & 1,1 & 0 & 0,7 \\
Obesidade grau II & 0 & 0 & 0 \\
Obesidade grau III & 1,1 & 1,9 & 1,4 \\
\hline Prevalência geral & & & 0,16 \\
\hline Desnutridos & 50,5 & 62,3 & 54,7 \\
Não desnutridos & 49,5 & 37,7 & 45,3 \\
\hline Tese Qui-qu & & 0,7 \\
\hline
\end{tabular}

Teste Qui-quadrado

"Diferença significativa $(\mathrm{p}<0,05)$ 
A doença mais frequente entre os desnutridos foi a HAS (44,4\%), seguida de doenças endócrinas $(30,9 \%)$, outras $(30,9 \%)$, do trato gastrointestinal $(27,4 \%)$, do trato geniturinário $(23,5 \%)$, neoplásicas (21\%), respiratórias $(18,5 \%)$, hematológicas $(13,6 \%)$, cardiovasculares $(12,3 \%)$, reumáticas $(7,4 \%)$, autoimunes $(3,7 \%)$, do sistema nervoso $(3,7 \%)$, imunológicas $(1,4 \%)$ e neurológicas $(1,2 \%)$.

O número médio de doenças da amostra foi de 2,22 $\pm 1,16$. Não houve diferença estatística $(\mathrm{p}=0,38)$ entre número de doenças e estado nutricional. No entanto, quando o número médio de doenças foi comparado entre as duas diferentes faixas etárias, constatou-se que os idosos possuíam maior número de doenças associadas em comparação aos pacientes adultos ( $p<0,0001)$ (tabela 3).

Tabela 3. Número de doenças associadas de acordo com estado nutricional e faixa etária da população estudada na Clínica Médica Feminina e Masculina do Hospital de Clínicas da Universidade Federal do Paraná Curitiba-PR, 2013

\begin{tabular}{lcc}
\hline \multicolumn{1}{c}{ Estado nutricional $^{\mathrm{a}}$} & № de doenças associadas $( \pm \mathrm{DP})$ & $p$ \\
\hline Sem risco & $1,95 \pm 1,10$ \\
Desnutrição grave & $2,38 \pm 1,14$ \\
Desnutrição moderada & $2,28 \pm 1,17$ & 0,38 \\
Desnutrição leve & $2,43 \pm 1,19$ \\
Eutrofia & 3 \\
Sobrepeso & $3 \pm 2,82$ \\
Obesidade grau I & 3 \\
Obesidade grau II & 3 \\
Obesidade grau III & $1,97 \pm 1,14$ \\
\hline Faixa etáriab $^{\mathrm{b}}$ & $2,68 \pm 1,07$ \\
\hline Adultos & $2,22 \pm 1,16$ \\
Idosos $_{\text {Amostra geral }}$ & \\
\hline
\end{tabular}

${ }^{\text {aTeste ANOVA }}$

${ }^{b}$ Teste Mann-Whitney

"Diferença significativa $(\mathrm{p}<0,05)$ 
Em relação ao tempo de permanência hospitalar dos pacientes, a média da população geral foi de 14,68 \pm 12,76 dias de internação. A análise estatística demonstrou haver diferença significativa $(\mathrm{p}=0,03)$ entre as diferentes classificações do estado nutricional e tempo de permanência hospitalar (tabela 4). E em relação tempo de permanência hospitalar, não houve diferença estatística entre os pacientes desnutridos e não desnutridos $(p=0,47)$ (tabela 4$)$.

Tabela 4. Tempo de permanência hospitalar em relação ao estado nutricional da população estudada na Clínica Médica Feminina e Masculina do Hospital de Clínicas da Universidade Federal do Paraná Curitiba-PR, 2013

\begin{tabular}{lcc}
\hline & \multicolumn{2}{c}{ Tempo de permanência hospitalar } \\
\cline { 2 - 3 } \multicolumn{1}{c}{ Estado nutricional ${ }^{\mathrm{a}}$} & $(x$ dia s $\pm \mathrm{DP})$ & $p$ \\
\hline Sem risco & $14,31 \pm 11,63$ & \\
Desnutrição grave & $14,25 \pm 17,47$ & $0,03^{*}$ \\
Desnutrição moderada & $10,32 \pm 6,75$ & \\
Desnutrição leve & $16,3 \pm 11,38$ \\
Eutrofia & 12 & \\
Sobrepeso & $24 \pm 16,97$ & 0,47 \\
Obesidade grau I & 14 & \\
Obesidade grau II & - & \\
Obesidade grau III & $43,5 \pm 48,79$ \\
\hline Desnutridos & $14,05 \pm 11,87$ \\
Não desnutridos & $15,43 \pm 13,81$ & \\
Amostra geral & $14,68 \pm 12,76$ & \\
\hline
\end{tabular}

aTeste ANOVA

${ }^{b}$ Teste Mann-Whitney

"Diferença significativa $(\mathrm{p}<0,05)$

A frequência de óbitos ocorridos entre os pacientes foi de 5,4\%. Dentro deste índice de mortalidade, todos eram pacientes adultos, representando $8,4 \%$ da população adulta total e havendo diferença estatística $(p=0,03)$ em comparação à população idosa. Entre os indivíduos que foram a óbito, 25\% possuíam algum grau de desnutrição; não houve, entrentanto, diferença estatística entre mortalidade e desnutrição $(p=0,14)$. 


\section{Discussão}

As doenças do trato geniturinário, cardiovasculares, hipertensão arterial sistêmica e neoplásicas foram mais frequentes nos pacientes idosos em comparação aos adultos. Essas doenças, somadas às osteoarticulares e respiratórias crônicas, acometem a população acima dos 60 anos, aumentando a probabilidade de morte e diminuindo a expectativa de vida livre da incapacidade. ${ }^{17}$

O Estudo Nutricional da América Latina (ELAN) avaliou o estado nutricional de 9.348 pacientes internados em hospitais distribuídos pela América Latina, através da Avaliação Subjetiva Global (ASG). A desnutrição esteve presente em 50,2\% dos pacientes. Trinta e três por cento daqueles que estavam internados há apenas dois dias estavam desnutridos, e aqueles internados há 14 dias ou mais eram 59,7\%, representando uma piora do estado nutricional dos pacientes durante o período da internação..$^{18}$

O Inquérito Brasileiro de Nutrição Hospitalar (IBRANUTRI), estudo transversal, multicêntrico e epidemiológico, realizado com uma amostra de quatro mil pacientes internados em hospitais brasileiros, constatou, a partir da ASG, que 48,1\% dos pacientes estavam desnutridos. Entre estes, $33,2 \%$ já possuíam esta condição nos dois primeiros dias de hospitalização. ${ }^{10}$

Comparativamente com os estudos supracitados, a frequência de pacientes desnutridos na admissão hospitalar da atual pesquisa foi superior. Vale destacar que houve diferença na metodologia empregada entre este estudo e os demais. Porém, se comparado com a prevalência de desnutridos ao longo da permanência hospitalar, os resultados são similares, tendo em vista que os pacientes podem desenvolver desnutrição durante a internação. No presente estudo, a prevalência de pacientes com desnutrição foi de 54,7\%; com sobrepeso, 1,4\%; e com obesidade, 2,1\%. Dados semelhantes foram encontrados em estudo que avaliou pacientes internados no período de 2008 a 2009, no mesmo serviço de saúde (Hospital de Clínicas da Universidade Federal do Paraná) e obteve como resultado que 45,7\% dos pacientes estavam desnutridos, $8,3 \%$ com sobrepeso e 4,8\% com obesidade, demonstrando poucas mudanças no perfil nutricional dos pacientes em quatro anos. ${ }^{7}$

Sabe-se que a desnutrição é o distúrbio nutricional importante mais observado nos idosos e ocorre por causas secundárias ao envelhecimento, como alterações fisiológicas e nutricionais, condições socioeconômicas, patologias e outros fatores clínicos relacionados. A desnutrição no idoso causa consequências significativas ao paciente e piora o estado mórbido em geral. ${ }^{15,19}$ Entretanto, nesta pesquisa, não houve diferença significativa entre a frequência de desnutrição das faixas etárias estudadas, assim como no estudo realizado por McWhirter e Pennington, ${ }^{20}$ que observaram desnutrição na admissão hospitalar em pacientes de cirurgia geral, clínica geral, medicina respiratória, cirurgia ortopédica e unidades de avaliação de medicina para idosos. Os resultados encontrados eram similares e distribuídos homogeneamente entre os diferentes grupos, sugerindo, assim como no presente estudo, que a desnutrição pode ser um fator independente da idade. 
A hipertensão arterial sistêmica e doenças endócrinas - diabetes mellitus sendo a mais frequente (81\% - dados não apresentados) - foram as doenças mais frequentes, tanto na amostra geral, como na amostra dos desnutridos. O padrão de ocorrência de doenças se modificou nas últimas quatro décadas, por meio dos processos de transições demográficas, epidemiológicas e nutricionais na população mundial, prevalecendo, assim, o crescente aumento das doenças crônicas não transmissíveis, que se somam às causas externas, responsáveis por dois terços da carga de doenças no Brasil..11,22

Na revisão sistemática quantitativa, realizada por Pereira et al. ${ }^{23}$ entre 2003 e 2008, considerando 44 estudos em 35 países, foi revelada uma prevalência média global de 37,8\% dos homens e $32,1 \%$ das mulheres hipertensos.

Assim como a hipertensão arterial sistêmica, a diabetes mellitus também afeta a população mundial de forma preocupante. Segundo a Federação Internacional de Diabetes, 382 milhões de pessoas vivem com diabetes atualmente, e em 2035 este número aumentará 55\% ${ }^{24}$ Portanto, diante dos antecedentes científicos e índices globais, verifica-se coerência nos resultados, que revelam alta prevalência dessas doenças na população estudada, devido ao crescente aumento na população mundial.

Seguido da hipertensão e doenças endócrinas, as patologias mais comuns entre pacientes desnutridos foram, respectivamente, doenças do trato gastrointestinal, do trato geniturinário e neoplásicas.

A associação entre neoplasias e o estado nutricional tem sido estudada desde que Shields Warren propôs que a desnutrição era a principal causa de morte em indivíduos com câncer. ${ }^{25}$ As neoplasias contribuem para a piora do estado nutricional, assim como diversos estudos demonstram, ${ }^{3,14,26}$ além da perda espontânea de apetite, alterações no paladar e no olfato, perda de peso involuntária e anorexia, que pode estar relacionada à própria doença, ao desenvolvimento da caquexia, medicamentos e sintomas gastrointestinais. ${ }^{27}$

Os sintomas gastrointestinais como anorexia, náuseas, distensão e dor abdominal também são causas da redução de ingestão alimentar, perda de peso involuntária e piora do estado nutricional nos pacientes diagnosticados com doenças relacionadas ao trato gastrointestinal, além de alterações no metabolismo de nutrientes, digestão e absorção, causando a esses pacientes, maior propensão à desnutrição. ${ }^{14,28}$

Os dados sobre tempo médio de permanência hospitalar e a mortalidade dos pacientes foram analisados, a fim de verificar possíveis relações com a presença ou ausência de desnutrição nos pacientes. Os dados encontrados sugerem que os pacientes diagnosticados com obesidade grau III permaneceram mais tempo internados. Verificou-se, porém, que os pacientes desnutridos não ficaram mais tempo internados em comparação aos pacientes não desnutridos. Assim como os óbitos e a faixa etária que também não sofreram influência da desnutrição. 
O impacto da desnutrição no aumento do tempo de permanência hospitalar foi abordado da mesma maneira no estudo realizado por Thomas et al. ${ }^{29}$, no qual também foi verificada ausência de diferença estatística significativa entre essas variáveis. No entanto, outros estudos ${ }^{10,11,30}$ demonstram que pacientes com desnutrição têm seu tempo de internação prolongado e maiores taxas de mortalidade, comparada a pacientes não debilitados nutricionalmente. Isto ocorre pois a desnutrição afeta a função respiratória, pela consequente perda muscular, aumenta a susceptibilidade de infecções, reduz a função cardíaca, prejudica o sistema imunológico e o estado metabólico geral. ${ }^{18}$

A equipe de nutrição, portanto, se torna essencial já que possui papel de suporte à terapia médica, sendo capaz de retardar o processo catabólico proteico e falência de órgãos. ${ }^{31}$ A partir do prévio diagnóstico de desnutrição, torna-se viável a implementação da terapia nutricional adequada, a fim de manter ou recuperar o estado nutricional do paciente e evitar a instalação ou progressão da desnutrição e de suas complicações. ${ }^{32}$

\section{Conclusão}

Verificou-se significativa frequência de pacientes com desnutrição na admissão hospitalar. No entanto, com relação às variáveis analisadas, tempo de permanência hospitalar e mortalidade, estas não sofreram influência da presença de desnutrição.

Seria oportuna a realização de futuros estudos com objetivos similares, de caráter longitudinal, que acompanhassem o processo de internação dos pacientes, desde a admissão até a alta hospitalar, possibilitando a análise de outros fatores e variáveis decorrentes da permanência dos pacientes no hospital.

\section{Referências}

1. Silva SRJ, Waitzberg DL. Gasto energético. In: Waitzberg DL. Nutrição oral, enteral e parenteral na prática clínica. $3^{a}$ ed. São Paulo: Atheneu; 2002. p. 327-342.

2. Leandro-Merhi VA, Garcia RWD, Tafner B, Florentino MC, Casteli R, Aquino JLB. Relação entre o estado nutricional e as características clínicas de pacientes internados em enfermaria de cirurgia. Rev. Ciênc. Méd. 2000; 9(3):105-114.

3. Leandro-Merhi VA, Mônaco DV, Lazarini ALG, Yamashiro A, Maciel AC. Estado nutricional de pacientes internados em um hospital privado. Rev. Bras. Nutr. Clin. 2004; 19(3):116-122.

4. Beghetto MG. Estado nutricional como preditor de morte, infecção e permanência hospitalar [tese]. [Porto Alegre]: Universidade Federal do Rio Grande do Sul, Faculdade de Medicina; 2007.

5. Saunders J, Smith T. Malnutrition: causes and consequences. Clinical Medicine 2010; 10(6):624-627. 
6. Bollet AJ, Owens S. Evaluation of nutritional status of selected hospitalized patients. Am. J. Clin. Nutr. 1973; 26(9):931-938.

7. Ordoñez AM, Schieferdecker MEM, Cestonaro T, Cardoso Neto J, Campo ACL. Nutritional status influences the length of stay and clinical outcomes in hospitalized patients in internal medicine wards. Nutr. Hosp. 2013; 28(4):1313-1320.

8. Támer GL, López MDR, Cruz AJP. Hospital malnutrition: relation between the hospital length of stay and the rate of early readmissions. Med. Clin. (Barc). 2009; 132(10):377-84.

9. Borghi R, Meale MMS, Gouveia MAP, França JID, Damião AOMC. Perfil nutricional de pacientes internados no Brasil: 19222 pacientes (Estudo BRAINS). Rev. Bras. Nutr. Clin. 2013; 28(4):255-63.

10. Waitzberg DL, Caiaffa WT, Correia MITD. Hospital Malnutrition: The Brazilian National Survey (IBRANUTRI): a study of 4000 patients. Nutrition 2001; 17(7/8):573-580.

11. Warnold I, Lundholm K. Clinical significance of preoperative nutritional status in 215 noncancer patients. Ann. Surg.1984; 199(3):299-305.

12. Waitzberg DL. Nutriçao enteral e parenteral na pratica clinica. $2^{\mathrm{a}}$ ed. Atheneu; 1995.

13. Correia MITD, Waitzberg DL. The impact of malnutrition on morbidity, mortality, length of hospital stay and costs evaluated through a multivariate model analysis. Clin. Nutr. 2003; 22(3):235-239.

14. Rezende IFB, Oliveira VS, Kuwano EA, Leite APB, Rios I, Dórea YSS, et al. Prevalência da desnutrição hospitalar em pacientes internados em um hospital filantrópico em Salvador (BA), Brasil. R. Ci. Méd. Biol. 2004; 3(2):194-200.

15. Kyle UG, Unger P, Mensi N, Genton L, Pichard C. Nutrition status in patients younger and older than $60 \mathrm{y}$ at hospital admission: a controlled population study in 995 subjects. Nutrition 2002; 18(6):463-9.

16. Kondrup J, Allison SP, Elia M, Vellas B, Plauth M. ESPEN Guidelines for Nutrition Screening 2002. Clinical Nutrition 2003; 22(4):415-421.

17. Campolina AG, Adami F, Santos JLF, Lebrão ML. A transição de saúde e as mudanças na expectativa de vida saudável da população idosa: possíveis impactos da prevenção de doenças crônicas. Cad. Saúde Pública 2013; 29(6):1217-1229.

18. Correia MITD, Campos ACL. Prevalence of hospital malnutrition in latin america: The Multicenter ELAN Study. Nutrition 2003; 19(10):823-825.

19. Stratton RJ, King CL, Stroud MA, Jackson AA, Elia M. Malnutrition universal screening tool predicts mortality and length of hospital stay in acutely ill elderly. Br. J. Nutr. 2006; 95:325-330.

20. McWhirter JP, Pennington CR. Incidence and recognition of malnutrition in hospital. BMJ 1994; 308(6934):945-948.

21. Toscano CM. As campanhas nacionais para detecção das doenças crônicas não-transmissíveis: diabetes e hipertensão arterial. Ciênc. Saúde Coletiva 2004; 9(4):885-895.

22. Malta DC, Cezário AC, Moura L, Morais Neto OL, Silva Junior JB. A construção da vigilância e prevenção das doenças crônicas não transmissíveis no contexto do Sistema Único de Saúde. Epidemiol. Serv. Saúde 2006; 15(1):47-65. 
23. Pereira M, Lunet N, Azevedo A, Barros H. Differences in prevalence, awareness, treatment and control of hypertension between developing and developed countries. J. Hypertens. 2009; 27(5):963-75.

24. International Diabetes Federation. IDF Diabetes Atlas. Sixth Edition. Bruxelas: IDF; 2013.

25. Guimarães GC. Nutrição e câncer. Acta Oncol. Bras. 2002; 22(1):227-232.

26. Kruizenga HM, Wierdsma NJ, Bokhorst MA, van der Schueren, Haollander HJ, Jonkers-Schuitema CF, et al. Screening of nutritional status in The Netherlands. Clin. Nutr. 2003; 22(2):147-52.

27. Silva MPN. Síndrome da anorexia-caquexia em portadores de câncer. Rev. Bras. Cancerologia 2008; 52(1):59-77.

28. Sena FG, Taddeo EF, Andrade Neto ER, Ferreira MSR, Rolim EG. Estado nutricional de pacientes internados em enfermaria de gastroenterologia. Rev. Nutr. 1999; 12(3):233-239.

29. Thomas JM, Isenring E, Kellett E. Nutritional status and length of stay in patients admitted to an Acute Assessment Unit. J. Hum. Nutr. Diet. 2007; 20(4):320-8.

30. Goiburu ME, Goiburu MM, Bianco H, Díaz JR, Alderete F, Palacios MC, et al. The impact of malnutrition on morbidity, mortality and length of hospital stay in trauma patients. Nutr. Hosp. 2006; 21(5):604-610.

31. Maicá AO, Schweigert D. Nutritional assessment of severely ill patient. Rev. Bras. Ter. Intensiva 2008; 20(3):286-295.

32. Garcia RWD, Leandro-Merhi VA, Pereira AM. Estado nutricional e sua evolução em pacientes internados em clínica médica. Rev. Bras. Nutr. Clin. 2004; 19(2):59-63. 
